\title{
Hodgkin's Paragranuloma Involving Spleen
}

National Cancer Institute

\section{Source}

National Cancer Institute. Hodgkin's Paragranuloma Involving Spleen. NCI Thesaurus.

Code C26961.

An obsolete term that refers to Hodgkin lymphoma with splenic involvement. 